\title{
Investigation of Dopamine Analogues: Synthesis, Mechanistic Understanding, and Structure-Property Relationship
}

\author{
Huamin $\mathrm{Hu},{ }^{\dagger}$ Jason Christopher Dyke, ${ }^{\dagger}$ Brett Allen Bowman, ${ }^{\dagger}$ Ching-Chang Ko, ${ }^{\ddagger}$ and Wei You* ${ }^{\dagger}, \S$ \\ ${ }^{\dagger}$ Department of Chemistry, University of North Carolina at Chapel Hill, Chapel Hill, North Carolina 27599-3290, United States \\ ${ }^{\ddagger}$ Department of Orthodontics and Applied Materials Sciences Program, University of North Carolina at Chapel Hill, Chapel Hill, \\ North Carolina 27599-7450, United States \\ ${ }^{\S}$ Department of Applied Physical Sciences, University of North Carolina, Chapel Hill, North Carolina 27599-3216, United States
}

\section{Supporting Information}

ABSTRACT: Dopamine, perhaps the simplest molecule that covalently links catechol and amine, together with its derivatives, has shown impressive adhesive and coating properties with its polymers. However, the scope of the molecules is rather limited, and the polymerization mechanisms are still elusive. We designed a general synthetic scheme and successfully synthesized a series of dopamine analogues with different alkyl chain lengths between the catechol and amine. Taking these new dopamine analogues, together with the molecular systems that have separate catechol and alkyl amine, we show that having both catechol and amine in the molecular system, whether covalently linked via an alkyl chain or not, is sufficient to polymerize under a similar reaction condition to that of dopamine polymerization. However, the timedependent UV-vis characterization of the individual polymerization indicates that the polymerization for individual molecular systems likely proceeds via different reaction intermediates, depending on the length of the alkyl chain and whether there is a covalent linkage. Interestingly, whereas the covalent linkage via an alkyl chain is not necessary for showing the adhesive property, it is required to achieve the impressive coating property. Our results offer new insights into the design and synthesis of dopamine analogues for future applications, as well as a further mechanistic understanding of the polymerization of these dopamine analogues.

\section{INTRODUCTION}

The unique moisture-resistant adhesion of marine mussels has attracted scientists' attention in recent years. ${ }^{1}$ Studies revealed that the mussel-adhesion is mediated by five unique adhesive proteins, all of which contain a significant amount of a catecholcontaining amino acid, L-3,4-dihydroxyphenylalanine (LDOPA), and amine-containing amino acids such as lysine, histidine, and arginine. ${ }^{2}$ Unsurprisingly, the fundamental understanding of the functions of these mussel adhesive proteins $s^{2,3}$ has facilitated the design of synthetic small molecules/polymers to mimic the unique adhesive property of mussels. ${ }^{4-6}$ Reciprocally, investigating these mussel proteininspired small molecules/polymers, in particular, the structureproperty relationship, has helped to identify the key functional components and the working mechanism for these unique adhesion/coating behaviors. Pioneering work by the Deming group $^{7}$ demonstrated that the catechol functionality of L-DOPA is the primary component required to replicate the moistureresistant adhesion. Further studies have shown that catechol and its oxidized $o$-quinone form are largely responsible for the adhesive behavior through hydrogen bonding 8,9 and crosslinking via metal chelating ${ }^{9,10}$ as well as aryl-aryl coupling, ${ }^{4,11}$ respectively. ${ }^{12}$ These discoveries have promoted the incorpo- ration of catechol and its derivatives into various polymer backbones to achieve functional hydrogels and adhesives, among others. ${ }^{4,5,13-17}$

Although the importance of having the catechol functional group to reproduce the mussel's adhesive property has been generally agreed upon, the potential involvement of the amine group in achieving the mussel's adhesive property has been much less studied. Researchers suggested that amine may contribute to the mussel's adhesion via ionic bonding to negatively charged surfaces ${ }^{18,19}$ and intermolecular cross-linking with $o$-quinones through Michael addition or Schiff-base formation. ${ }^{9}$ Thus, having both catechol and amine would create a synergistic effect to reproduce the mussel's adhesive properties. $^{20}$ Indeed, in 2007, the Messersmith group ${ }^{21}$ first reported that dopamine, a small molecule that covalently links both catechol and amine, could form polydopamine (PDA) under mild conditions, e.g., under a buffer condition of $\mathrm{pH}$ 8.5. ${ }^{21}$ More impressively, PDA demonstrated an almost universal adhesion/coating property to a variety of substrates,

Received: June 8, 2016

Revised: August 15, 2016

Published: September 5, 2016 
Scheme 1. Overview of Mussel-Inspired Materials and Proposed Polymer Structure

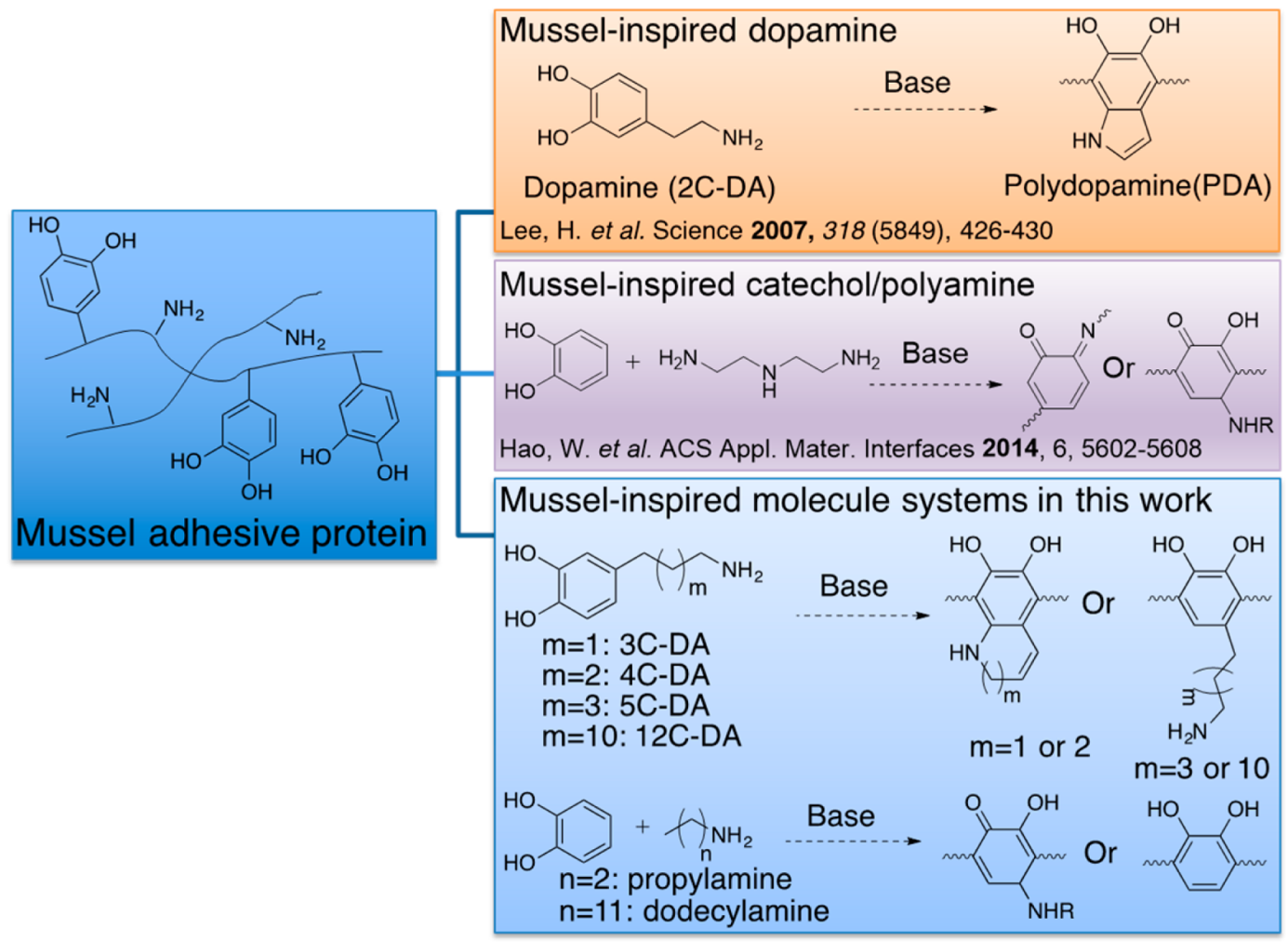

including metals, ceramics, and polymers, among others. ${ }^{21}$ Inspired by the formation mechanism of the eumelanin polymer, Messersmith and co-workers proposed a similar polymerization mechanism to explain the formation of PDA (Scheme 1). This proposed mechanism first involves the oxidation of dopamine to dopaminequinone, which can then cyclize to form leukodopaminechrome. Subsequent oxidation and rearrangement would form the premonomer that can then polymerize via oxidative coupling into the PDA. ${ }^{21}$ Though a generally agreed formation mechanism of PDA and its exact chemical structure remain elusive, ${ }^{2-25}$ the easy synthesis of PDA and its impressive coating/adhesive property have promoted the application of PDA and its derivative materials in various fields, including energy, environment, and biomedical engineering. ${ }^{6}$

However, PDA and its related materials have their own limitations. For instance, the dark-brown/black color associated with PDA and its related materials ${ }^{21}$ are not desirable for certain clinical applications. Further structural functionalization of dopamine molecule without impacting its polymerization behavior also appears to be difficult; the only reported strategy to covalently incorporate dopamine with other polymers/ organic materials is through its amine group, which, for example, can form amide with the pendent carboxylic acid from the parent polymer. ${ }^{16,17,26,27}$ This status quo motivated us to explore new mussel-adhesive-inspired molecules that could maintain notable features of dopamine/PDA, including watersolubility, facile polymerization, and an extraordinary coating ability while mitigating issues in current systems.

On the basis of all of these previous studies, we hypothesized that any molecule that covalently links the catechol and primary amine functional groups would likely show polymerization and coating behavior similar to those demonstrated by dopamine/ PDA. However, the polymerization mechanism could be molecule-dependent, in particular when factoring in the proposed ring-formation process in the dopamine polymerization. In general, forming a cyclic product from a linear precursor would be affected by both entropy and enthalpy. For example, in the series of dopamine analogues shown in Scheme 1, 3C-DA and 4C-DA would likely undergo cyclization during their polymerization, leading to the formation of cyclic structures (e.g., six-membered ring for 3C-DA) in their polymers, similar to the proposed five-membered ring formation in the case of dopamine (i.e., 2C-DA). This is because five- and six-membered rings are the most stable cyclics. Indeed, an earlier computational study of the intramolecular amine addition to ortho-quinones ${ }^{28}$ indicated the possibility of forming six- and seven-membered rings. On the other hand, it is hard to imagine that 5C-DA and 12C-DA, having longer alkyl chains, would be able to undergo cyclization to form an eight-membered ring (for 5C-DA) and a larger ring structure (for 12C-DA). This is because the large ring formation via intramolecular ring closure is entropically disfavored. Therefore, having all of these new dopamine analogues and investigating their polymerization behaviors would help shed more light on the polymerization mechanisms of dopamine and its analogues. Furthermore, comparing the adhesive and coating properties shown by these dopamine analogues would help to disclose how these subtle structural changes (i.e., the length of the alkyl chain between the catechol and the amine) would influence the adhesive/coating ability of PDA and its derivative materials.

Thus, the objective of our study is threefold: synthesis, mechanistic investigation, and structure-property relationship. We find that all of these new dopamine analogues are able to polymerize, albeit likely via different mechanisms, based on experimental observations. Although 3C-DA and 4C-DA could go through similar polymerization pathways (e.g., cyclization 
Scheme 2. Synthesis of the 3C-DA Analogue

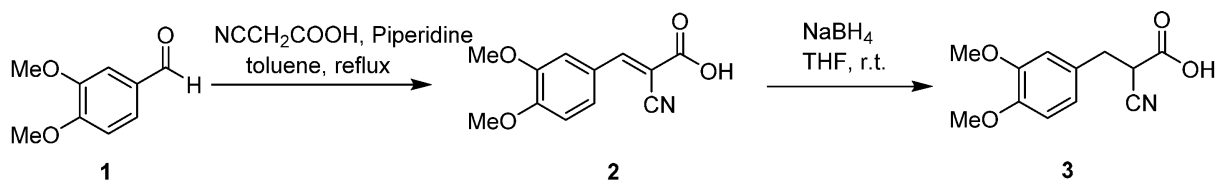
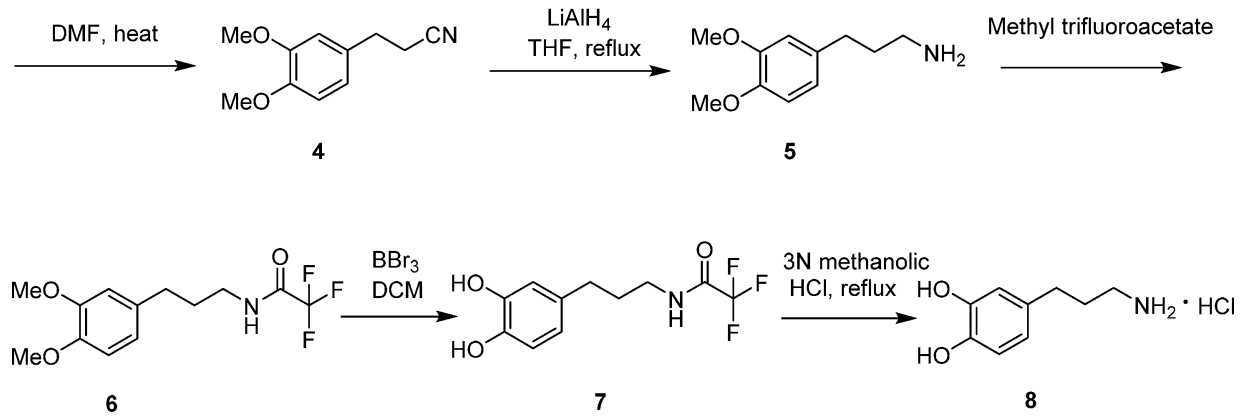

Scheme 3. Synthesis of 4C-DA, 5C-DA, and 12C-DA Analogues<smiles>COc1ccccc1OC</smiles>
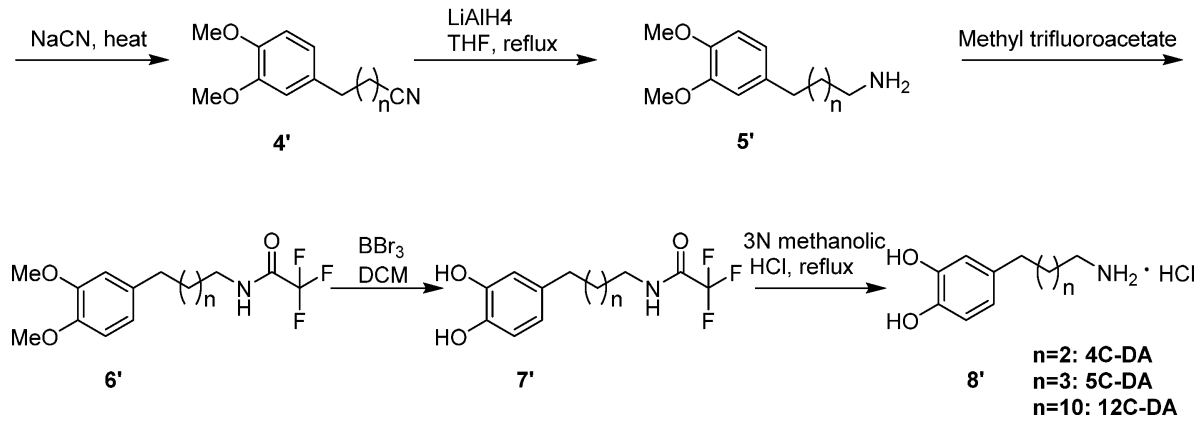

followed by oxidative coupling) to the one proposed for dopamine (Scheme 1), 5C-DA and 12C-DA appear to go through a simpler reaction pathway to polymerize (e.g., without cyclization). Importantly, the adhesive properties of polymers from these dopamine analogues (including dopamine) are tightly correlated with the number of catechol and amine functionalities in the system, with a negligible influence from the length of the alkyl chain that links catechol and amine. Furthermore, even the alkyl linkage between catechol and amine does not seem to be a prerequisite for achieving the adhesive property. In fact, noncovalently linked catechol and amine-based molecular systems (e.g., catechol and "free" propyl amine), when subjected to the same polymerization condition, also form materials that show comparable shear adhesive strength to those achieved with covalently linked catechol and amine via an alkyl chain. On the other hand, this covalent linkage via an alkyl chain seems to be critical in achieving the coating ability of these analogous catechol-amine systems: although the covalently linked catechol and amine systems also show comparable coating ability with their polymers to that of PDA, materials formed with unlinked catechol and amine show only mediocre coating abilities.

\section{MATERIALS AND METHODS}

Material and Substrate Preparation. Dopamine hydrochloride (99\%), 3,4-dimethoxybenzealdehyde (99+\%), cyanoacetic acid (99\%), sodium borohydride $\left(\mathrm{NaBH}_{4}, 99 \%\right.$, powder), triethylamine (98\%), and lithium alumina hydride (LAH, 95\%, powder) were purchased from Acros Organics and used as received. Piperidine (99\%), methyl trifluoroacetate (99\%), $1 \mathrm{M}$ boron tribromide in methylene chloride (DCM), and $3 \mathrm{~N}$ methanolic $\mathrm{HCl}$ solution were purchased from Sigma-Aldrich and used as received. Hexanes, acetone, DCM, toluene, methanol $(\mathrm{MeOH})$, ethyl acetate (EtOAc), tetrahydrofuran (THF), and dimethylformamide (DMF) were obtained from Fisher. THF was freshly distilled over sodium before use. Poly(ethylene terephthalate) (PET), poly(tetrafluoroethylene) (PTFE), glass, and indium tin oxide (ITO) were cleaned ultrasonically in 2-propanol (IPA) for 15-20 min before use. A gold (20 nm deposited onto $5 \mathrm{~nm} \mathrm{Ti}$ ) surface on $\mathrm{Si}$ wafers was prepared by electron beam deposition.

Characterization Methods. ${ }^{1} \mathrm{H}$ and ${ }^{13} \mathrm{C}$ NMR spectra were recorded on Bruker AC-400 (400 MHz) spectrometers. $\mathrm{CDCl}_{3}$ and DMSO- $d_{6}$ were used as the solvent. Mass spectra were acquired at the University of North Carolina, Chapel Hill mass spectrometry facility. UV-vis absorbance spectra were obtained on a UV-2600 spectrophotometer (Shimadzu Scientific Instruments, Inc.). Lap-shear testing was conducted on an Instron 5566. For the coating experiments, different substrates were vertically immersed in a diluted aqueous solution of a given molecular system (e.g., $2 \mathrm{mg}$ of dopamine or 3C-DA per 

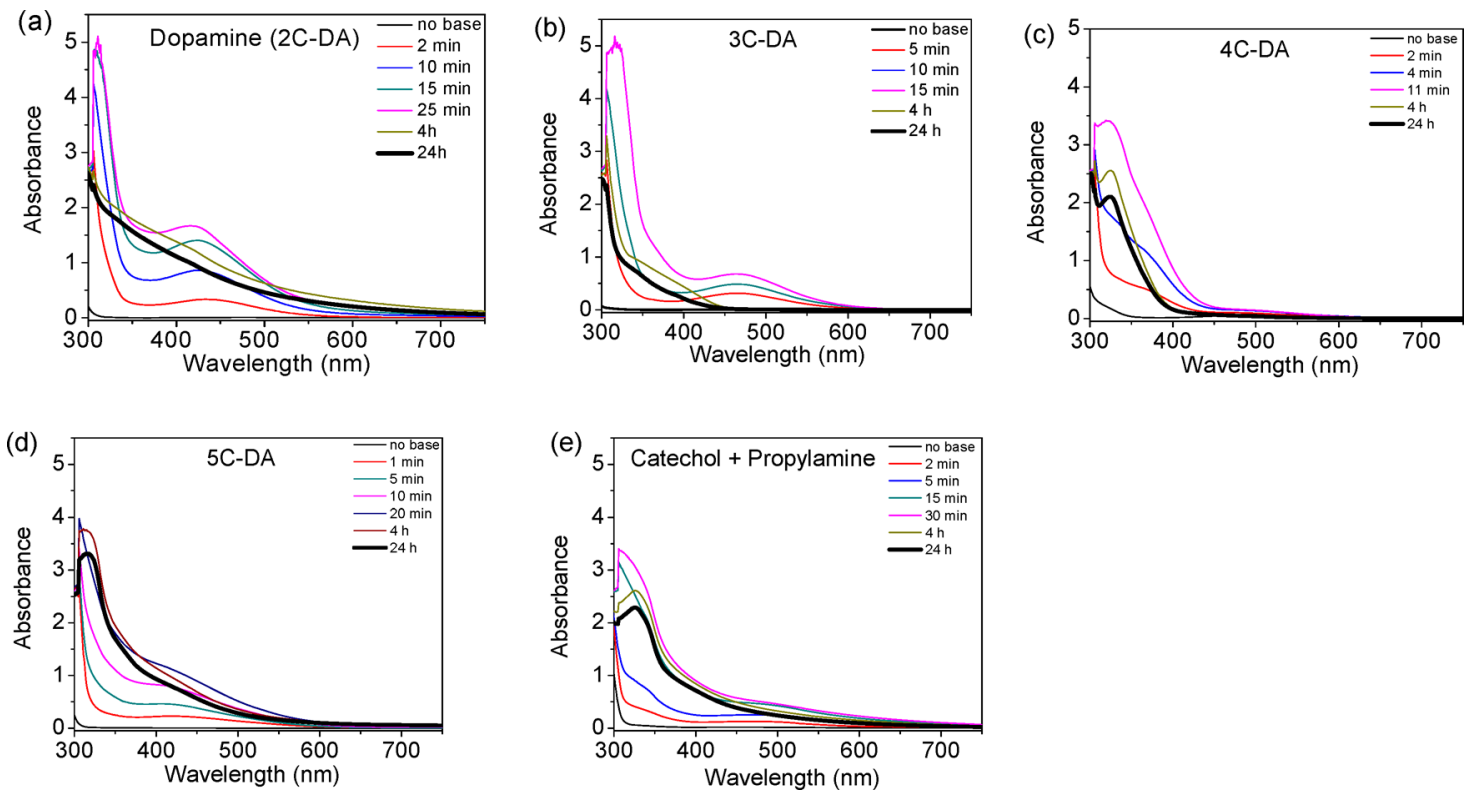

Figure 1. Time-dependent UV-vis spectroscopy for the polymerization of (a) dopamine, (b) 3C-DA, (c) 4C-DA, (d) 5C-DA, and (e) catechol and propylamine in aqueous solution after adding $1 \mathrm{M} \mathrm{NaOH}$.

milliliter of $10 \mathrm{mM}$ Tris buffer, $\mathrm{pH} 8.5$ ) with gentle stirring for $17 \mathrm{~h}$. The substrates were then washed with distilled water and dried over nitrogen gas. X-ray photoelectron spectroscopy (XPS) was conducted on a Kratos Axis Ultra DLD X-ray photoelectron spectrometer using a monochromatic $\mathrm{Al} \mathrm{K} \alpha$ source $(1486.8 \mathrm{eV})$.

\section{RESULTS AND DISCUSSION}

Synthesis of Dopamine Analogues. Because of the high reactivity of dopamine analogues that have unprotected amine and catechol, all of these analogues were synthesized in their $\mathrm{HCl}$ salt form, similar to the commercially available dopamine (also as its $\mathrm{HCl}$ salt). Scheme 2 outlines the synthesis route that successfully offered the 3C-DA in its $\mathrm{HCl}$ salt form. A condensation of commercially available 3,4-demethoxybenzaldehyde with cyanoacetic acid, followed by $\mathrm{NaBH}_{4}$ reduction, leads to compound 3 . The decarboxylation of 3 gives 3-(3,4dimethoxyphenyl)propylnitrile (4), which undergoes another reduction by $\mathrm{LiAlH}_{4}$ to afford key intermediate 3-(3,4dimethoxyphenyl) propylamine (5). ${ }^{29}$ Multiple attempts to remove the methyl from the methoxy group (e.g., via $\mathrm{BBr}_{3}$ treatment) to directly convert 5 to the targeted 3C-DA failed, possibly because of the interference of the primary amine functionality of $\mathbf{5}$. Thus, we decided to protect the primary amine with a trifluoroacetyl ( $\mathrm{Tfa}$ ) group, converting 5 into 6, following a similar protocol previously reported. ${ }^{30}$ Treating 6 with the strong Lewis acid $\mathrm{BBr}_{3}$ can then smoothly remove these two methyl groups, revealing the catechol of 7. In the last step, the Tfa protecting group is readily cleaved under strong acidic condition to generate the target molecule (3C-DA) in its $\mathrm{HCl}$ salt form 8 . The chemical structure of the product formed after each step was confirmed by ${ }^{1} \mathrm{H}$ NMR and ${ }^{13} \mathrm{C}$ NMR, respectively, and the chemical formula of target molecule 8 was further verified by ESI-MS.

The rather general synthesis of dopamine analogues with longer alkyl linkers in between the catechol and amine (4C-DA, $5 \mathrm{C}-\mathrm{DA}$, and $12 \mathrm{C}-\mathrm{DA})$ is largely similar to that of 3C-DA; the major difference lies in the preparation of 3-(3,4dimethoxyphenyl)alkylnitrile, molecule $\left(4^{\prime}\right)$ in Scheme 3. Specifically, a Friedel-Crafts acylation of the commercially available 3,4-dimethoxybenzene $\left(\mathbf{1}^{\prime}\right)$, followed by a $\mathrm{NaBH}_{4}$ reduction, readily affords $3^{\prime}$. A nucleophilic substitution of the bromide in $3^{\prime}$ with the cyano offers $4^{\prime}$, which is the structural analogue to 4 in Scheme 2. Molecule $4^{\prime}$ can then go through similar reactions outlined in Scheme 2 (i.e., from 4 to 8 ) to afford targeted molecule $\mathbf{8}^{\prime}$, also in its $\mathrm{HCl}$ salt form.

Polymerization Behavior Studied by UV-Vis Spectra. After having obtained a substantial quantity of these dopamine analogues (in their $\mathrm{HCl}$ form), we next explored the polymerization behavior of these newly prepared dopamine analogues, together with dopamine $\cdot \mathrm{HCl}$ as the control/ reference. Given the difficulty in separating reaction intermediates from the polymerization, we chose to monitor the polymerization process by UV-vis spectroscopy, which has been previously used to track the polymerization progress of dopamine. ${ }^{31,32}$ Though Tris buffer has been a popular medium for the polymerization of dopamine, we have noticed that Tris buffer could be incorporated into the PDA structure, ${ }^{23}$ which could interfere with our study on the polymerizations. Thus, we simplified the polymerization condition and carried out the polymerization of different dopamine analogues in aqueous solution with excess $\mathrm{NaOH}$ base. ${ }^{33}$ Through comparing the time-dependent UV-vis absorption spectra during the polymerization to the absorption feature of known reaction intermediates, ${ }^{13,31,32}$ we can identify plausible reaction pathways.

Experimentally, after adding an excess amount of base (dopamine analogues: $1 \mathrm{M} \mathrm{NaOH}=1: 8$, molar ratio) to a dilute aqueous solution $(1 \mathrm{mM})$ of dopamine/3C-DA/4C-DA/5CDA (12C-DA is not soluble in water), the UV-vis absorbance spectra were recorded at different time intervals for $24 \mathrm{~h}$. Figure la shows the time-dependent UV-vis spectra of the polymerization of dopamine, which agrees well with a previous literature report. ${ }^{32}$ The most notable feature of the UV-vis spectra is the appearance of an absorbance peak at $420 \mathrm{~nm}$ immediately after the base addition, which gradually develops its intensity in the first $25 \mathrm{~min}$ and then attenuates. As the polymerization progresses, this absorption peak broadens and gradually decreases, and the whole spectrum becomes almost 

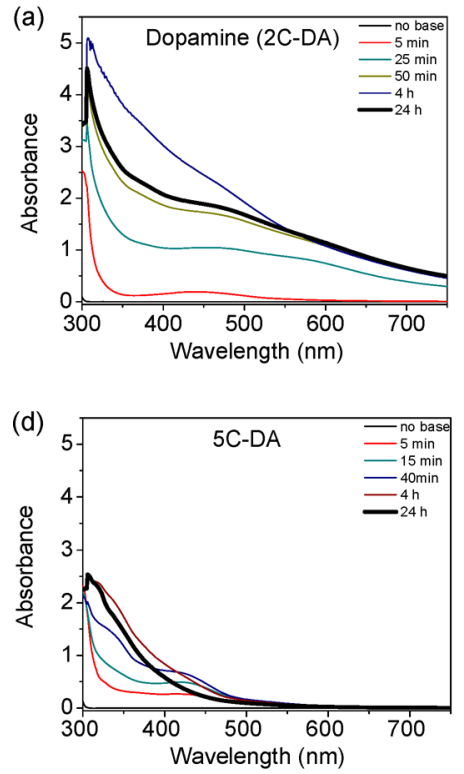
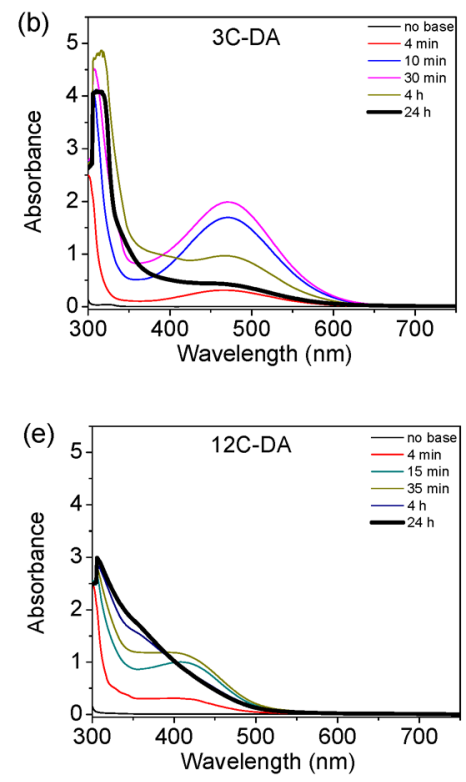
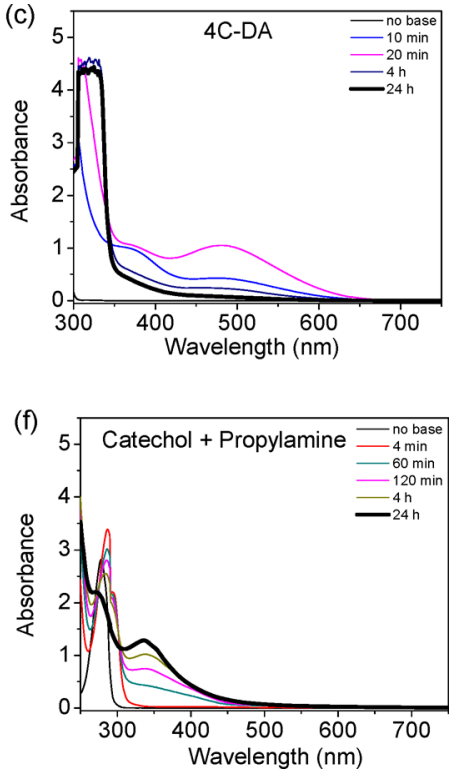

Figure 2. Time-dependent UV-vis spectroscopy for (a) dopamine, (b) 3C-DA, (c) 4C-DA, (d) 5C-DA, (e) 12C-DA, and (f) catechol and propylamine in $\mathrm{MeOH}(1 \mathrm{mM})$ after adding $1 \mathrm{M} \mathrm{NaOH}(\mathrm{MeOH} /$ water $=125: 1, \mathrm{v} / \mathrm{v})$.

featureless but widely absorptive after $4 \mathrm{~h}$, which explains the black/brown color of polydopamine (PDA). Similar timedependent UV-vis spectra for the polymerization of 3C-DA was observed (Figure 1b); however, the absorption peak position shifts to $470 \mathrm{~nm}$, and the maximum peak intensity ever reached is much lower than that of the $420 \mathrm{~nm}$ absorption peak observed in the dopamine polymerization. Nevertheless, the absorption spectrum loses this absorption peak $(\sim 470 \mathrm{~nm})$ as the polymerization proceeds. More interestingly, the spectrum after $4 \mathrm{~h}$ does not bear much intensity after $450 \mathrm{~nm}$, hence the solution containing poly(3C-DA) appears almost colorless (at low concentration) or much lighter in color (at high concentration) than the solution of poly(2C-DA) (i.e., PDA). Interestingly, with one more methylene in its structure than 3C-DA, 4C-DA does not show any obvious absorption peaks during its polymerization, even at the very beginning (i.e., 2 $\mathrm{min}$ ). This could be caused by a fast polymerization rate, which would render the observation of the reaction intermediate more difficult, given the transient nature of such a reaction intermediate. Similar to 3C-DA, 4C-DA almost loses its absorption intensity entirely in the range of 400 to $600 \mathrm{~nm}$ during its polymerization (Figure 1c), whereas 5C-DA regains its absorption in the same region in the UV-vis spectrum (Figure 1d). Finally, the polymerization of catechol and propylamine (i.e., the noncovalently linked molecular system of 3C-DA) also has noticeable absorption within the range of 400 to $600 \mathrm{~nm}$ (Figure 1e), though no clearly defined peak can be identified in the same range. Nevertheless, in all cases, the UV-vis absorption spectrum becomes almost featureless after $24 \mathrm{~h}$, with much less intensity in the visible region than that of the spectrum from the polymerization of dopamine.

Given that an alcohol-water mixed solvent has been used to affect the polymerization rate of dopamine ${ }^{34,35}$ and $12 \mathrm{C}-\mathrm{DA}$ is insoluble in water but has better solubility in methanol $(\mathrm{MeOH})$, we next chose methanol as the solvent to conduct the polymerization and aimed to spectroscopically capture the reaction intermediates. After adding the same excess amount of base (dopamine analogues: $1 \mathrm{M} \mathrm{NaOH}=1: 8$ molar ratio) to a dilute $\mathrm{MeOH}$ solution $(1 \mathrm{mM})$ of dopamine/3C-DA/4C-DA/
5C-DA/12-DA, we again monitored their polymerization by UV-vis (Figure 2). A few interesting observations can be noted. First, the noticeable absorption peak at $420 \mathrm{~nm}$ in the early stage of the dopamine polymerization in water (Figure 1a) loses its intensity for the same polymerization in the methanol solution (Figure 2a), though a hump at $470 \mathrm{~nm}$ can still be identified in this broad absorption. Second, the UV-vis spectrum for the 3C-DA polymerization in the mixed solvent (Figure $2 \mathrm{~b}$ ) is qualitatively similar to that in water (Figure $1 \mathrm{~b}$ ). However, the $470 \mathrm{~nm}$ peak has shown a higher intensity, and the time it takes to reach the highest absorbance intensity is twice that in water. Interestingly, the 4C-DA polymerization in the mixed solvent develops a new absorption peak at around $480 \mathrm{~nm}$, which eventually disappears after $24 \mathrm{~h}$ (Figure 2c). If we assume that the $\sim 470(480) \mathrm{nm}$ absorption peak indicates the formation of the polymerization intermediate, then a shorter time to reach the maximum intensity and a faster disappearance of this absorption peak would imply more rapid formation of the polymer (Figures S1 and S2 in Supporting Information). Under this assumption, it appears that the polymerization of either 3C-DA or 4C-DA in the methanol solution $(\mathrm{MeOH} /$ water $=125: 1, \mathrm{v} / \mathrm{v})$ is slower than the corresponding polymerization in water. Third, the dopamine analogues with longer aliphatic linkers (5C-DA and 12C-DA) show qualitatively similar time-dependence UV-vis spectra (Figure 2d,e), with a noticeable absorption peak at $410 \mathrm{~nm}$ in the early stage of the polymerization. Nevertheless, in all of these polymerizations of dopamine and its analogues in the mixed solvent, the UV-vis spectra do not contain identifiable absorption peaks beyond $350 \mathrm{~nm}$ after $24 \mathrm{~h}$, similar to what we observed for the polymerizations in water. In contrast, the UVvis spectra for the noncovalently linked catechol/propylamine have developed an absorption peak at $326 \mathrm{~nm}$, whose intensity continues to rise even after $24 \mathrm{~h}$ (Figure $2 \mathrm{f}$ ). Increasing the alkyl chain length for the noncovalently linked system has a negligible impact on the polymerization, as evidenced by the fact that almost identical UV-vis spectra were observed for catechol/dodecylamine (Figure S2g in Supporting Information). 


\section{Scheme 4. Proposed Reaction Intermediates during Polymerization}
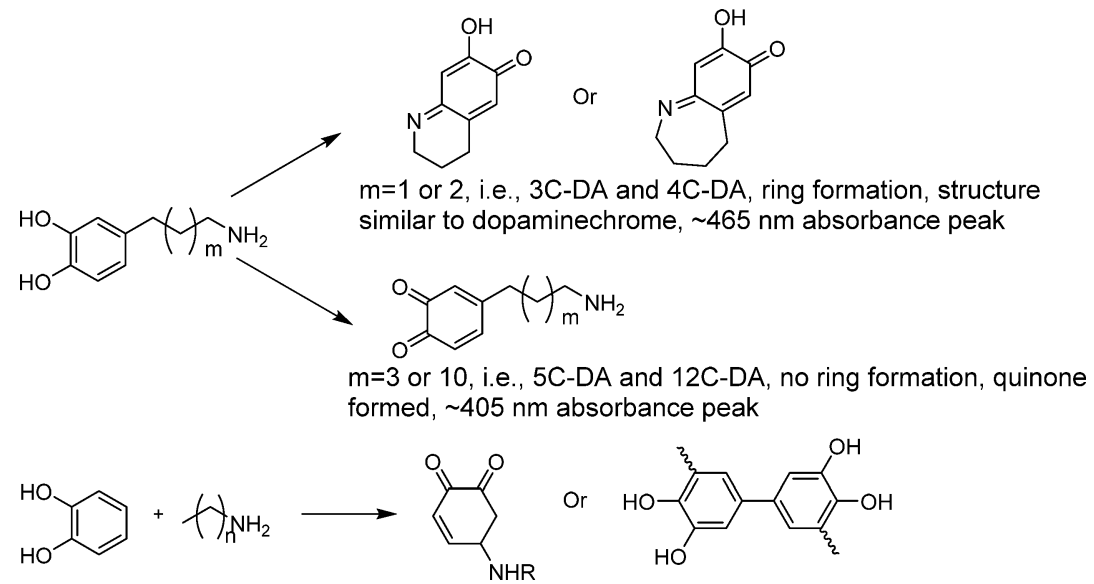

$\mathrm{n}=2$ or 11 , i.e., catechol/propylamine or catechol/dodecylamine no ring formation, polycatechols or Michael addition product $\sim 320 \mathrm{~nm}$ absorbance peak

Combining our time-dependent UV-vis data in both $\mathrm{H}_{2} \mathrm{O}$ and $\mathrm{MeOH}$ with literature proposals on the polymerization mechanism of dopamine, ${ }^{22-25}$ we propose plausible mechanisms for the observed polymerizations as follows. First, the absorbance peak at $\sim 470 \mathrm{~nm}(480 \mathrm{~nm}$ ) (Figures $1 \mathrm{~b}$ and $2 \mathrm{~b}, \mathrm{c}$ ), appearing in the early stage of the polymerization of $3 \mathrm{C}-\mathrm{DA}$ and 4C-DA agrees well with the absorbance of dopachrome, which has been previously identified during the biosynthesis of eumelanin from L-DOPA and has a $\lambda_{\max }$ of $473 \mathrm{~nm}^{13}$ Therefore, we tentatively assign this peak at $470 \mathrm{~nm}(480$ $\mathrm{nm})$ to the formation of 3C-dopachrome and 4C-dopachrome (Scheme 4) via a similar formation process for dopachrome (i.e., intramolecular cyclization followed by oxidation). On the other hand, for entropic reasons, it would be difficult for similar cyclization occurring during the polymerization of $5 \mathrm{C}-\mathrm{DA}$ or 12C-DA, both having longer aliphatic chains between the catechol and the amine. Thus, we tentatively ascribe the 410 $\mathrm{nm}$ absorption peak that appeared during the 5C-DA and $12 \mathrm{C}$ $\mathrm{DA}$ polymerization (Figure $2 \mathrm{~d}, \mathrm{e}$ ) to the presence of $5 \mathrm{C}$ dopaquinone and 12C-dopaquinone (Scheme 4). In fact, this $410 \mathrm{~nm}$ peak agrees well with the $\sim 400 \mathrm{~nm}$ absorption peak for dopaquinone. ${ }^{11}$ In all cases (3C- to $12 \mathrm{C}$-DA), the intensity of the absorption peak would decrease after a short period of time because cross-coupling between the structural units of catechol and/or quinone would increasingly dominate the reaction progress to form the polymer (Scheme S3 in Supporting Information).

However, for dopamine polymerization, the UV-vis spectrum is more complicated. First, in $\mathrm{H}_{2} \mathrm{O} / \mathrm{NaOH}$, we did not observe any absorbance peak at $\sim 470 \mathrm{~nm}$ (Figure 1a), which was believed to indicate the formation of dopaminechrome, a known reaction intermediate for dopamine polymerization. This could be caused by the high $\mathrm{pH}(8 \mathrm{mM} \mathrm{NaOH})$ and thereby rapid spectral evolution, which could mask the initial $\sim 470 \mathrm{~nm}$ peak. ${ }^{36}$ Furthermore, we speculate that the observed unique absorption peak at $\sim 420 \mathrm{~nm}$ (Figure 1a), which has also been observed by others, ${ }^{32}$ might indicate the formation of some oligomers (e.g., coupling of phenols) in the early stage of dopamine polymerization. Interestingly, when we decreased the polymerization rate for dopamine polymerization with $\mathrm{MeOH} / \mathrm{H}_{2} \mathrm{O}(\mathrm{v} / \mathrm{v}=125: 1)$, we observed a broad absorption over the entire spectral range (Figure 2a) with a hump near $470 \mathrm{~nm}$, which implies the formation of dopaminechrome. Nevertheless, the broad absorption that rendered the dark color of PDA is believed to be a result of its complicated, presumably cross-linked structure analogous to that of eumelanin. ${ }^{21}$ It is very likely that PDA is a mixture of oligomers/polymers with various monomeric components (e.g., dopamine, dihydroxyindole, indoledione, etc.), tied together via cross-linking and noncovalent interactions. ${ }^{24,25}$ In contrast, the much lighter color of these new dopamine analogues after polymerization, supported by the highly attenuated absorption in the visible region after $24 \mathrm{~h}$ (Figure $2 \mathrm{~b}-\mathrm{e}$ ), suggests simpler structures that have less conjugation than PDA. Given the structural similarity between dopamine and these dopamine analogues, further investigation of the polymerization mechanism of these dopamine analogues-ideally, together with other dopamine analogues-can offer more insights into a clearer picture of the formation of PDA.

On the other hand, when the amine is not covalently linked with the catechol, the UV-vis spectra of polymerizations with these molecular systems (i.e., catechol/propylamine and catechol/dodecylamine) are distinctly different from those observed with covalently linked ones (Figure $2 \mathrm{f}$ and Figure S2g in Supporting Information vs Figure $2 \mathrm{a}-\mathrm{e}$ ). In particular, the absorption peak at $326 \mathrm{~nm}$ gains more intensity as polymerization continues and becomes much more visible after $24 \mathrm{~h}$. We tentatively assign this absorbance peak at $326 \mathrm{~nm}$ to the Michael addition product between the quinone and amine or phenol coupling (Scheme 4). The polymerization of such noncovalently linked systems could undergo coupling among semiquinone and/or quinones as well as products from Michael addition to form the polymers. ${ }^{37}$

Finally, please note that the proposed mechanisms are largely postulated on the basis of the time-dependent UV-vis absorption data for the studied molecular systems in the mixed solvent $(\mathrm{MeOH} /$ water $=125: 1, \mathrm{v} / \mathrm{v})$. The noticeable difference in UV-vis spectra for the same molecular system in different solvents implies that the polymerization mechanism could also depend on the given solvent system, in particular, the water-dominant system vs the methanol-dominant system.

Adhesive Property Studied by Lap-Shear Testing. Many mussel-inspired materials have shown significant adhesive properties; ${ }^{5,14,21}$ because dopamine and the analogues in this 

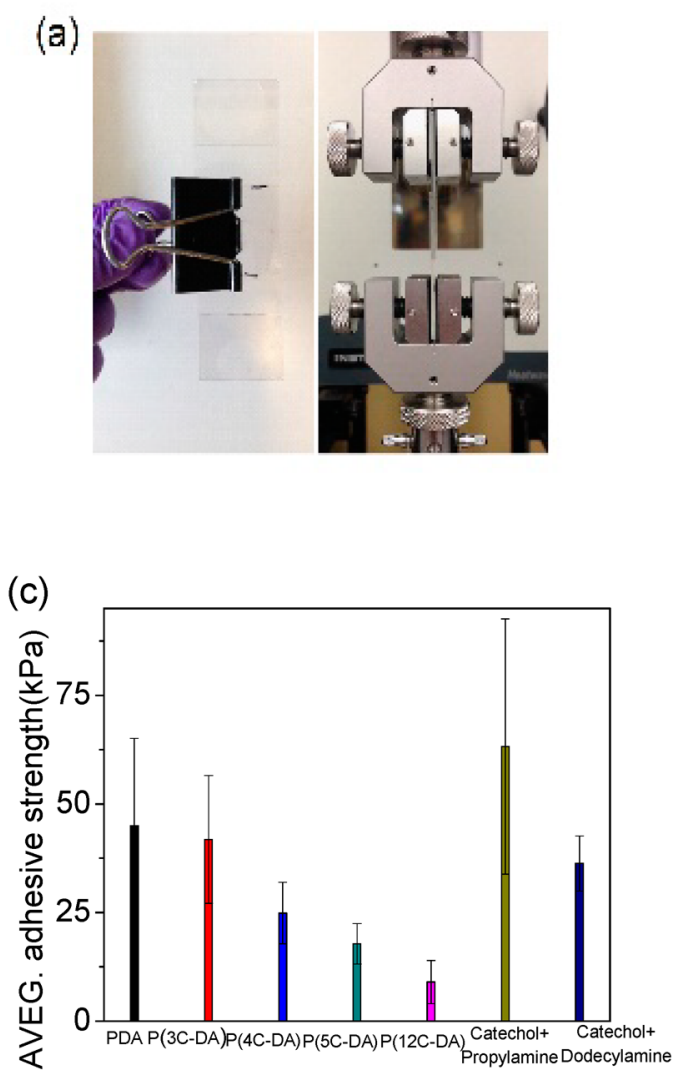

(b)

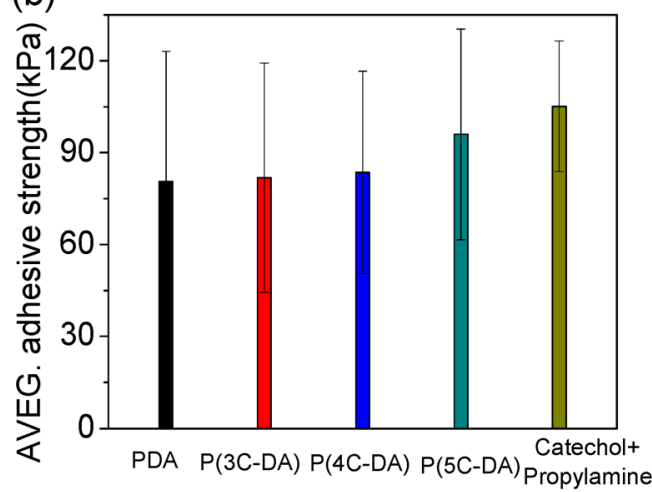

(d)

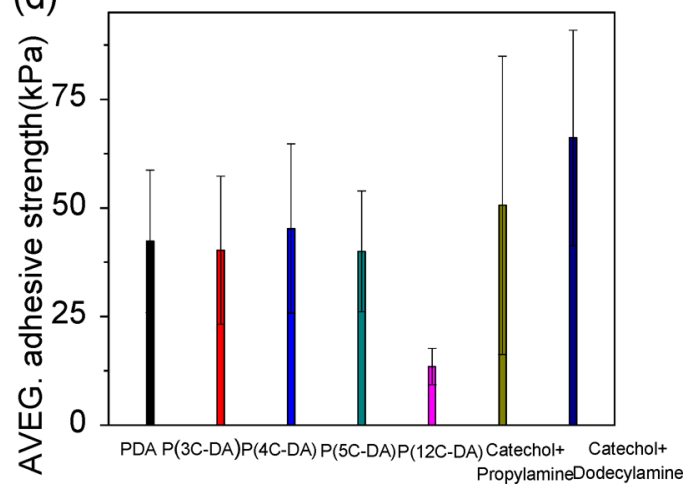

Figure 3. (a) Image of test sample (left) and main part of the Instron machine (right) for lap-shear testing. (b) Results for adhesive strength of PDA, poly(3C-DA), poly(4C-DA), poly(5C-DA), and catechol/propylamine in water at $6.7 \mathrm{mg} / \mathrm{mL}$. (c) Results for adhesive strength of PDA, poly(3CDA), poly(4C-DA), poly(5C-DA), catechol/propylamine, and catechol/dodecylamine in IPA: $\mathrm{H}_{2} \mathrm{O}$ at $6.7 \mathrm{mg} / \mathrm{mL}\left(\mathrm{IPA}: \mathrm{H}_{2} \mathrm{O}=1: 2\right.$, v/v). (d) Results for adhesive strength of PDA, poly(3C-DA), poly(4C-DA), poly(5C-DA), catechol/propylamine, and catechol/dodecylamine in IPA: $\mathrm{H}_{2} \mathrm{O}$ at 33.3 $\mathrm{mM}\left(\mathrm{IPA}: \mathrm{H}_{2} \mathrm{O}=1: 2, \mathrm{v} / \mathrm{v}\right)$.

study are also inspired by mussels, we next investigated the bulk adhesive properties of all molecular systems in our study. Specifically, we measured the lap shear adhesion of two glass substrates sandwiching the polymer, following a protocol established by the Wilker group. ${ }^{14}$ We first dissolved some monomers (including 2C-DA, 3C-DA, 4C-DA, 5C-DA, and catechol/propylamine) in water or all monomers (2C-DA, 3CDA, 4C-DA, 5C-DA, 12C-DA, catechol/propylamine, and catechol/dodecylamine) in isopropanol/water $(1: 2, \mathrm{v} / \mathrm{v})$. Please note that isopropanol (IPA) was used here instead of methanol in order to mitigate the solubility issue of $12 \mathrm{C}-\mathrm{DA}$. In fact, these two solvent systems appear to offer similar polymers via similar polymerization mechanisms for each studied molecular system because UV-vis spectra for the polymerization in the mixed solvent where $\mathrm{H}_{2} \mathrm{O}$ was dominant (IPA$\mathrm{H}_{2} \mathrm{O}, \mathrm{v} / \mathrm{v}=1: 2$ ) (Figure S3 in Supporting Information) are very comparable to those obtained in $\mathrm{H}_{2} \mathrm{O}$ (Figure $\mathrm{S} 1$ in Supporting Information), for each studied molecular system.

We then added excess base $(100 \mu \mathrm{L} 1 \mathrm{M} \mathrm{NaOH}$ aqueous solution) to induce the polymerization. After $5 \mathrm{~min}$, we applied $30 \mu \mathrm{L}$ of the polymer solution to one side of the glass slide, which was then covered with another identical glass slide with an overlapping area of $1 \mathrm{in.} \times 1 \mathrm{in}$. A binder clip was applied to maintain this sandwich structure for the next $48 \mathrm{~h}$ to ensure the completion of the polymerization (Figure 3a, left). This binder clip was removed before the sandwich structure was tested via an Instron (Figure 3a, right). After measuring the maximum loads at the break point for each polymer, the adhesion strength was calculated by the maximum load/sandwich area (i.e., 1 in. $\times 1$ in. in our case). A load vs extension curve was obtained for each sample, which was qualitatively similar to literature results. $^{14}$ (Figure S8 in Supporting Information), indicating the complete removal of the solvent. In addition, we measured the UV-vis spectra of the polymers sandwiched in between the glass substrates. The UV-vis spectrum of the polymer does not contain characteristic absorption features of the reaction intermediate (for each studied system), and the overall spectrum is very similar to the final UV-vis spectrum (i.e., measured after $24 \mathrm{~h}$ ) of the same molecular system obtained in $\mathrm{H}_{2} \mathrm{O}$. These results confirmed that it was the polymers that were tested in the lap shear testing (Figure S9 in Supporting Information).

As shown in Figure 3b, polydopamine (i.e., PDA), poly(3C$\mathrm{DA})$, poly(4C-DA), and poly(5C-DA) have demonstrated comparable adhesive strength $(\sim 90 \mathrm{kPa})$ from identical polymerization in water (i.e., the same mass concentration of $6.7 \mathrm{mg} / \mathrm{mL}$ ), indicating that the increment of the aliphatic chain length between the amine and the catechol only negligibly influences the adhesive property of the polymer. Interestingly, the polymer from reacting catechol/propylamine under the same condition (i.e., catechol and propylamine together at $6.7 \mathrm{mg} / \mathrm{mL}$ in a $1: 1$ molar ratio) demonstrated almost identical adhesion strength to that of covalently linked dopamine analogues, implying that the covalent bonding (i.e., the alkyl linkage) between the amine and the catechol is not necessary to achieve the adhesive strength of the polymer. 
(a)

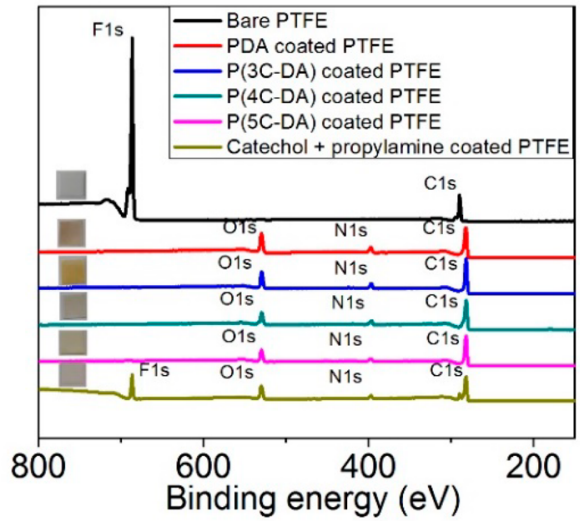

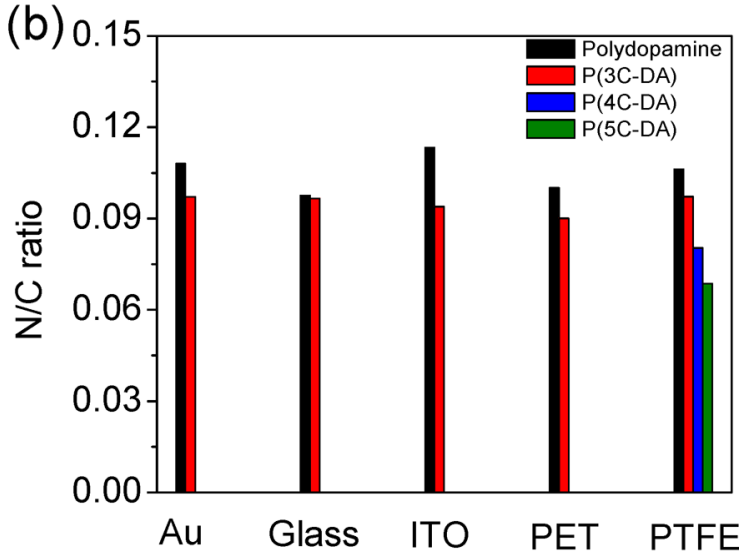

Figure 4. (a) XPS spectra of bare PTFE substrates, PDA-coated PTFE and poly(3C-DA)-coated PTFE, poly(4C-DA)-coated PTFE, and poly(5CDA)-coated PTFE. (b) Quantitative analysis of the nitrogen/carbon (N/C) ratio of different substrates coated with PDA and poly(3C-DA) as indicated by the black and red bars, respectively. Blue and green bars represent the N/C ratio of PTFE substrate coated with poly(4C-DA) and poly(5C-DA), respectively.

Again, because $12 \mathrm{C}$-DA is not soluble in water, we switched to the mixed solvent, IPA/water $(1: 2, \mathrm{v} / \mathrm{v})$. To compare with the results obtained with polymers synthesized in water, we used the same mass concentration $(6.7 \mathrm{mg} / \mathrm{mL})$ in the mixed solvent as well. Surprisingly, for each dopamine and its analogues with longer aliphatic chains, the adhesive strength of the polymer created in the mixed solvent is significantly smaller than that of the counterpart synthesized in water. We offer two plausible explanations. First, the mixed solvent (IPA/ $\mathrm{H}_{2} \mathrm{O}, \mathrm{v} / \mathrm{v}=1: 2$ ), which has a different solvent evaporation rate that that of pure $\mathrm{H}_{2} \mathrm{O}$, might lead to incomplete film formation under our experimental conditions. Alternatively, pure $\mathrm{H}_{2} \mathrm{O}$ may generate more polymers than the mixed solvent of IPA: $\mathrm{H}_{2} \mathrm{O}$ (though the structure of the polymers could be identical, regardless of the solvent system, as we previously discussed). Furthermore, the adhesive strength of the polymer decreases monotonically from dopamine to 12C-DA (Figure $3 c)$. However, when the same polymerizations were carried out with an identical molar concentration (i.e., $33 \mathrm{mM}$ ) in the mixed solvent, the adhesive strength from the different polymers became comparable (Figure 3d), except for the polymer from 12C-DA. We tentatively ascribe the weaker adhesive strength demonstrated by the polymer from 12C-DA in the mixed solvent to its limited solubility (even in the mixed solvent), which would significantly impede its polymerization. Nevertheless, these results indicate that the amount of catechol and amine in the system is proportional to the adhesive strength of polymers formed from these dopamine analogues in the mixed solvent. This conclusion is further supported by the fact that the polymers created from the noncovalently linked analogues (i.e., catechol/propylamine and catechol/dodecylamine) also show comparable adhesive strength to that of their covalently linked counterparts, when the same molar concentration $(33 \mathrm{mM})$ was used for the polymerization in the mixed solvent (Figure 3d). This observation also supports the previous conclusion that the covalent bonding between the amine and the catechol is not a prerequisite for achieving the adhesive strength of the polymer.

Coating Property Studied by XPS. The most impressive property of polydopamine (PDA) is its ability to coat almost any substrate, effectively serving as an adlayer to change the surface property of the coated substrate and allow further functionalization. Thus, our last task was to investigate the coating ability of these newly prepared polymers. To compare the coating ability of our new synthesized dopamine analogues to that of PDA, we followed a protocol reported earlier by the Messersmith group (i.e.,10 mM Tris buffer, $\mathrm{pH} \mathrm{8.5).}{ }^{21}$ To investigate whether the Tris buffer solution would have any impact to the polymerization when compared to the $\mathrm{NaOH}$ solution, we conducted a similar time-dependent UV-vis study of these dopamine analogs in the Tris buffer solution. Comparing the additional data (Figure S4 in Supporting Information) with the UV-vis data acquired in $\mathrm{H}_{2} \mathrm{O} / \mathrm{NaOH}$ (Figure S1 in Supporting Information), we did not discern any significant difference. Thus, we believe that the polymerization mechanisms (and related polymers) under the Tris buffer condition would be comparable to those under the $\mathrm{H}_{2} \mathrm{O}$ / $\mathrm{NaOH}$ condition.

We chose PTFE, which is known for its antifouling ability, as our target to study the coating ability of these polymers. Experimentally, the PTFE substrate was immersed in dilute aqueous solutions of 3C-DA, 4C-DA, 5C-DA, and catechol/ propylamine (i.e., $2 \mathrm{mg} / \mathrm{mL}$ in $10 \mathrm{mM}$ Tris buffer, $\mathrm{pH} 8.5$ ), which were then gently stirred overnight. A control experiment with dopamine $\cdot \mathrm{HCl}$ was also carried out in parallel after it was coated with the polymer (Figure 4a). The PTFE substrate coated with PDA has a black brown/gray color, and the ones coated with poly(3C-DA), poly(4C-DA), and poly(5C-DA) have lighter colors. More importantly, X-ray photoelectron spectroscopy (XPS) characterization of the substrates coated with these dopamine analogues reveals the complete absence of the signals specific to the substrate (e.g., fluorine) after the coating, demonstrating a shielding effect similar to that shown by $\mathrm{PDA}^{21}$ (Figure $4 \mathrm{a}$ and Figure $\mathrm{S} 11$ in Supporting Information). Further chemical analyses (via XPS) of the surface of these PTFE substrates after coating with these new polymers disclose that the nitrogen/carbon ratio is very close to the theoretical value of each dopamine analogue (Figure $4 b$, rightmost column). This further supports a complete surface coating of the PTFE substrate by polymers from these dopamine analogues, similar to the coating ability of PDA. ${ }^{21}$ However, the noncovalently linked system (i.e., catechol and amine) is not able to achieve the complete coating of the PTFE substrate because the fluorine signal (from the PTFE substrate) 
is still present after the coating (Figure 4a). These results suggest that the covalent bonding between the amine and the catechol might play an important role in maximizing the coating ability of these dopamine analogues.

To further investigate whether the excellent coating ability on PTFE demonstrated by these new dopamine analogues, similar to that of PDA, is also universal as PDA has shown, ${ }^{21}$ we chose 3C-DA as the example to test the coating of its polymer on other substrates. For comparison, dopamine was also included in this set of experiments. Four different substrates having representative surface properties-gold, indium tin oxide (ITO), glass, and poly(ethyleneterephalate) (PET) were coated with poly(3C-DA) or PDA, following the same protocol as illustrated above. Not surprisingly, there is a visible change in the color of the substrate after it is coated with the polymer (i.e., PDA or poly(3C-DA); Figure S10a in Supporting Information). XPS characterization of the surface of these substrates after coating reveals the complete disappearance of substrate signals, demonstrating the comparable coating ability of poly(3C-DA) to that of PDA (Figure S10b in Supporting Information). In addition, the nitrogen/carbon ratio, ranging from 0.902 to 0.972 on these coated substrates, is very close to the theoretical value of 3C-DA (0.1111) (Figure 4b, red bar), suggesting complete surface coverage on all of these substrates by poly(3C-DA), similar to that achieved by PDA (Figure $4 \mathrm{~b}$, black bar). These results strongly suggest that 3C-DA, after polymerization, offers a similar coating ability and general applicability to those of dopamine/PDA.

\section{CONCLUSIONS}

Through a series of molecular systems that are structurally analogous to dopamine, we demonstrate that the intriguing polymerization behaviors of dopamine are not unique to dopamine; in fact, it appears that all molecular systems that contain catechol and amine, covalently linked via an alkyl chain or not, are able to polymerize. However, the exact polymerization mechanism for an individual molecular system appears to be dependent on (a) whether the catechol and amine are covalently linked via an alkyl chain and (b) the length of the alkyl chain that links the catechol and amine. Furthermore, the solvent system chosen to carry out the polymerization also seems to have an impact on the polymerization mechanism. It appears that conditions where water is dominant (i.e., IPA/ $\mathrm{H}_{2} \mathrm{O}, \mathrm{H}_{2} \mathrm{O}$, and Tris buffer) should offer similar polymers via a similar mechanism for each studied molecular system, whereas the polymerization mechanism in methanol is different. Further mechanistic studies on these molecular systems and other related systems are needed to elucidate the polymerization mechanisms.

Furthermore, our results indicate that the covalent attachment of catechol and amine (e.g., as in the case of dopamine) appears to have only a negligible influence on the adhesive property shown by polydopamine; almost all studied molecular systems show comparable adhesive strength when normalized with the amount of catechol and amine. However, such covalent linking between catechol and amine via an alkyl chain is crucial in reproducing the impressive coating ability that polydopamine has shown; noncovalently linked catechol and amine only show mediocre coating behaviors. All of these findings offer valuable guidelines in selecting and designing mussel adhesive protein-inspired materials for specific applications.

\section{ASSOCIATED CONTENT}

\section{Supporting Information}

The Supporting Information is available free of charge on the ACS Publications website at DOI: 10.1021/acs.langmuir.6b02141.

Proposed polymerization mechanism for dopamine and dopamine analogues, synthesis procedures of dopamine analogues, ${ }^{1} \mathrm{H}$ and ${ }^{13} \mathrm{C}$ NMR spectra, UV-vis spectra, adhesive data, coating images, and XPS data (PDF)

\section{AUTHOR INFORMATION}

\section{Corresponding Author}

*E-mail: wyou@unc.edu.

\section{Funding}

The work was supported, in part, by NIH/NIDCR R01DE022816.

\section{Notes}

The authors declare no competing financial interest.

\section{ACKNOWLEDGMENTS}

We acknowledge UNC CHANL for all of the XPS measurements.

\section{REFERENCES}

(1) Lee, B. P.; Messersmith, P. B.; Israelachvili, J. N.; Waite, J. H. Mussel-Inspired Adhesives and Coatings. Annu. Rev. Mater. Res. 2011, 41, 99-132.

(2) Silverman, H. G.; Roberto, F. F. Understanding Marine Mussel Adhesion. Mar. Biotechnol. 2007, 9, 661-681.

(3) Waite, J. H. The phylogeny and chemical diversity of quinonetanned glues and varnishes. Comp. Biochem. Physiol. B, Comp. Biochem. 1990, 97, 19-29.

(4) Sedó, J.; Saiz-Poseu, J.; Busqué, F.; Ruiz-Molina, D. CatecholBased Biomimetic Functional Materials. Adv. Mater. 2013, 25, 653701.

(5) Ye, Q.; Zhou, F.; Liu, W. Bioinspired catecholic chemistry for surface modification. Chem. Soc. Rev. 2011, 40, 4244-4258.

(6) Liu, Y.; Ai, K.; Lu, L. Polydopamine and Its Derivative Materials: Synthesis and Promising Applications in Energy, Environmental, and Biomedical Fields. Chem. Rev. 2014, 114, 5057-5115.

(7) Yu, M.; Deming, T. J. Synthetic Polypeptide Mimics of Marine Adhesives. Macromolecules 1998, 31, 4739-4745.

(8) Waite, J. H.; Andersen, N. H.; Jewhurst, S.; Sun, C. Mussel Adhesion: Finding the Tricks Worth Mimicking. J. Adhes. 2005, 81, 297-317.

(9) Lee, H.; Scherer, N. F.; Messersmith, P. B. Single-molecule mechanics of mussel adhesion. Proc. Natl. Acad. Sci. U. S. A. 2006, 103, 12999-13003.

(10) Fant, C.; Hedlund, J.; Höök, F.; Berglin, M.; Fridell, E.; Elwing, $\mathrm{H}$. Investigation of Adsorption and Cross-Linking of a Mussel Adhesive Protein Using Attenuated Total Internal Reflection Fourier Transform Infrared Spectroscopy (ATR-FTIR). J. Adhes. 2010, 86, 25-38.

(11) Yu, M.; Hwang, J.; Deming, T. J. Role of 1-3,4Dihydroxyphenylalanine in Mussel Adhesive Proteins. J. Am. Chem. Soc. 1999, 121, 5825-5826.

(12) Yang, J.; Cohen Stuart, M. A.; Kamperman, M. Jack of all trades: versatile catechol crosslinking mechanisms. Chem. Soc. Rev. 2014, 43, $8271-8298$

(13) Lee, B. P.; Dalsin, J. L.; Messersmith, P. B. Synthesis and Gelation of DOPA-Modified Poly(ethylene glycol) Hydrogels. Biomacromolecules 2002, 3, 1038-1047.

(14) Matos-Pérez, C. R.; White, J. D.; Wilker, J. J. Polymer Composition and Substrate Influences on the Adhesive Bonding of a 
Biomimetic, Cross-Linking Polymer. J. Am. Chem. Soc. 2012, 134, 9498-9505.

(15) Ryu, J. H.; Hong, S.; Lee, H. Bio-inspired adhesive catecholconjugated chitosan for biomedical applications: A mini review. Acta Biomater. 2015, 27, 101-115.

(16) Lee, S.-B.; González-Cabezas, C.; Kim, K.-M.; Kim, K.-N.; Kuroda, K. Catechol-Functionalized Synthetic Polymer as a Dental Adhesive to Contaminated Dentin Surface for a Composite Restoration. Biomacromolecules 2015, 16, 2265-2275.

(17) Wu, H.; Sariola, V.; Zhu, C.; Zhao, J.; Sitti, M.; Bettinger, C. J. Transfer Printing of Metallic Microstructures on Adhesion-Promoting Hydrogel Substrates. Adv. Mater. 2015, 27, 3398-3404.

(18) Suci, P. A.; Geesey, G. G. Influence of Sodium Periodate and Tyrosinase on Binding of Alginate to Adlayers of Mytilus edulis Foot Protein 1. J. Colloid Interface Sci. 2000, 230, 340-348.

(19) Olivieri, M. P.; Olivieri, M. P.; Wollman, R. M.; Hurley, M. I.; Swartz, M. F. Using conformational analysis to identify structurally conserved regions of MAP peptides that exhibit cellular attachment ability. Biofouling 2002, 18, 149-159.

(20) Maier, G. P.; Rapp, M. V.; Waite, J. H.; Israelachvili, J. N.; Butler, A. Adaptive synergy between catechol and lysine promotes wet adhesion by surface salt displacement. Science 2015, 349, 628-632.

(21) Lee, H.; Dellatore, S. M.; Miller, W. M.; Messersmith, P. B. Mussel-Inspired Surface Chemistry for Multifunctional Coatings. Science 2007, 318, 426-430.

(22) Dreyer, D. R.; Miller, D. J.; Freeman, B. D.; Paul, D. R.; Bielawski, C. W. Elucidating the Structure of Poly(dopamine). Langmuir 2012, 28, 6428-6435.

(23) Hong, S.; Na, Y. S.; Choi, S.; Song, I. T.; Kim, W. Y.; Lee, H. Non-Covalent Self-Assembly and Covalent Polymerization CoContribute to Polydopamine Formation. Adv. Funct. Mater. 2012, 22, 4711-4717.

(24) Della Vecchia, N. F.; Avolio, R.; Alfe, M.; Errico, M. E.; Napolitano, A.; d'Ischia, M. Building-Block Diversity in Polydopamine Underpins a Multifunctional Eumelanin-Type Platform Tunable Through a Quinone Control Point. Adv. Funct. Mater. 2013, 23, 1331-1340.

(25) Liebscher, J.; Mrówczyński, R.; Scheidt, H. A.; Filip, C.; Hădade, N. D.; Turcu, R.; Bende, A.; Beck, S. Structure of Polydopamine: A Never-Ending Story? Langmuir 2013, 29, 10539-10548.

(26) Lee, H.; Lee, Y.; Statz, A. R.; Rho, J.; Park, T. G.; Messersmith, P. B. Substrate-Independent Layer-by-Layer Assembly by Using Mussel-Adhesive-Inspired Polymers. Adv. Mater. 2008, 20, 16191623.

(27) Liu, Y.; Meng, H.; Konst, S.; Sarmiento, R.; Rajachar, R.; Lee, B. P. Injectable Dopamine-Modified Poly(ethylene glycol) Nanocomposite Hydrogel with Enhanced Adhesive Property and Bioactivity. ACS Appl. Mater. Interfaces 2014, 6, 16982-16992.

(28) Land, E. J.; Ramsden, C. A.; Riley, P. A. An MO study of regioselective amine addition to ortho-quinones relevant to melanogenesis. Tetrahedron 2006, 62, 4884-4891.

(29) Vincze, Z.; Bíró, A. B.; Csékei, M.; Timári, G.; Kotschy, A. The Palladium-Catalyzed Preparation of Condensed Tetracyclic Heterocycles and their Application to the Synthesis of rac-Mangochinine. Synthesis 2006, 2006, 1375-1385.

(30) Liu, Z.; Hu, B.-H.; Messersmith, P. B. Acetonide Protection of Dopamine for the Synthesis of Highly Pure N-docosahexaenoyldopamine. Tetrahedron Lett. 2010, 51, 2403-2405.

(31) Wei, Q.; Zhang, F.; Li, J.; Li, B.; Zhao, C. Oxidant-induced dopamine polymerization for multifunctional coatings. Polym. Chem. 2010, 1, 1430-1433.

(32) Du, X.; Li, L.; Li, J.; Yang, C.; Frenkel, N.; Welle, A.; Heissler, S.; Nefedov, A.; Grunze, M.; Levkin, P. A. UV-Triggered Dopamine Polymerization: Control of Polymerization, Surface Coating, and Photopatterning. Adv. Mater. 2014, 26, 8029-8033.

(33) Ju, K.-Y.; Lee, Y.; Lee, S.; Park, S. B.; Lee, J.-K. Bioinspired Polymerization of Dopamine to Generate Melanin-Like Nanoparticles Having an Excellent Free-Radical-Scavenging Property. Biomacromolecules 2011, 12, 625-632.
(34) Yan, J.; Yang, L.; Lin, M.-F.; Ma, J.; Lu, X.; Lee, P. S. Polydopamine Spheres as Active Templates for Convenient Synthesis of Various Nanostructures. Small 2013, 9, 596-603.

(35) Jiang, X.; Wang, Y.; Li, M. Selecting water-alcohol mixed solvent for synthesis of polydopamine nano-spheres using solubility parameter. Sci. Rep. 2014, 4, 6070.

(36) Herlinger, E.; Jameson, R. F.; Linert, W. Spontaneous autoxidation of dopamine. J. Chem. Soc., Perkin Trans. 2 1995, 2, $259-263$.

(37) Deming, T. J. Mussel byssus and biomolecular materials. Curr. Opin. Chem. Biol. 1999, 3, 100-105. 